\title{
Effet de la chimiothérapie sur la fonction testiculaire et ovarienne
}

\author{
C. Linassier , Ph. Colombat
}

Service d'Oncologie Médicale. CHRU Bretonneau, 2 boulevard Tonnellé, 37044 Tours Cedex.

\section{RESUME}

Les progrès en cancérologie permettent des guérisons fréquentes chez l'enfant et l'adulte jeune. L'oligo azoospermie et les troubles fonctionnels du spermatozoïde chez l'homme, l'aménorrhée et la ménopause précoce chez la femme sont fréquents et préserver la fertilité des patients doit être un soucis du thérapeute. Les alkylants sont fortement gonadotoxiques suivant une relation de dose-dépendance. D'autres drogues comme le cisplatine, les anthracyclines, les vincaalcaloïdes et les épipodophyllotoxines sont modérément gonadotoxiques tandis que les antimétabolites ont une toxicité gonadique mineure. La fertilité est plus altérée chez l'homme que chez la femme et diminue d'autant plus chez la femme qu'elle est proche de la ménopause. L'ovaire prépubertaire est moins sensible aux effets des chimiothérapies mais n'est pas totalement protégé. Aucune tératogénicité n'a pu être formellement attribuée à la chimiothérapie. Néanmoins une grossesse pendant une exposition à des agents cytotoxiques est fortement déconseillée et un délai de deux ans semble pouvoir être recommandé avant une conception.

Mots clés : grossesse, fertilité, chimiothérapie, cancer, leucémie.

\section{INTRODUCTION}

Les avancées thérapeutiques en oncologie permettent des guérisons chez un nombre important de patients traités par chimiothérapie. Les améliorations pronostiques concernent particulièrement l'enfant et l'adulte jeune. Les données du National Cancer Institute (NCI) montrent que $65 \%$ des enfants américains, qui ont été traités pour cancer entre 1983 et 1989, sont vivants plus de 5 ans après le traitement, contre seulement $28 \%$ il y a 20 ans [1]. En 1987, 125000 adultes américains avaient été traités pour cancer pendant leur enfance [2], et ce nombre est estimé à 200000 en l'an 2000 . Le développement des traitements adjuvants ne fait qu'accroître le nombre de patients qui sont exposés à des chimiothérapies. L'analyse des effets des chimiothérapies sur la fertilité s'avère néanmoins difficile si la fertilité est estimée en terme de grossesse menée à terme $[6,20]$. En effet, même en cas de fertilité conservée, une conception pourra être différée ou évitée par crainte d'une rechute, par inquiétude quant à la santé de l'enfant. La nécessité d'un recul important sur une grande cohorte de patients complique la méthodologie, si bien qu'aucune étude prospective n'a été publiée à ce jour.

\section{EFFETS DES CHIMIOTHERAPIES SUR LES ORGANES GENITAUX FEMININS}

Plusieurs séries de biopsies post-mortem chez des patientes traitées par chimiothéra- 
pie ont montré des altérations ovariennes variables en intensité selon les drogues administrées, les doses et l'association éventuelle à une irradiation [12]. Les lésions étaient habituellement générées par des polychimiothérapies, si bien qu'il était impossible de dresser la cartographie des lésions pour un seul médicament. Les atteintes comportaient à des degrés divers la réduction du volume ovarien, une atteinte corticale avec fibrose et parfois néovascularisation, une granulosa non proliférante, l'absence de maturation folliculaire, la réduction du nombre de follicules primordiaux, la rareté voire l'absence d'œuf, une augmentation des cicatrices atrétiques. Ces dommages étaient majorées par le mauvais état nutritionnel et le statu post-pubertaire [20]. L'ovaire pré-pubertaire n'est cependant pas totalement protégé contre les effets délétères de la chimiothérapie. Dans une série anatomopathologique $50 \%$ des fillettes traitées pour cancer présentaient des atteintes histologiques [20]. Les raisons de la résistance relative de l'ovaire prépubertaire aux antimitotiques demeurent obscures. Une réserve folliculaire importante ainsi qu'une réponse réduite à l'élévation des taux de FSH et de LH après chimiothérapie ont été évoquées [8], mais ne restent qu'au stade d'hypothèses.

L'atteinte de la granulosa se manifeste par des variations des taux de FSH, LH et d'œstradiol identiques à celles observées en période péri ménopausique. La stimulation de l'axe hypothalamo-hypophysaire est susceptible d'accélérer le recrutement folliculaire, augmentant ainsi la sensibilité ovarienne aux drogues cytotoxiques [8].

\section{EFFETS DE LA CHIMIOTHERAPIE SUR LES ORGANES GENITAUX MASCULINS}

Tous les stades de la spermatogenèse sont accessibles à la toxicité des antimitotiques. L'intensité des lésions est variable selon la nature et la posologie des produits utilisés.
Après survenue d'une azoospermie postthérapeutique, le repeuplement testiculaire suit la chronologie observée pendant la période pré-pubertaire. Selon la sévérité des atteintes de l'épithélium germinal et des cellules souches, l'azoospermie peut durer de quelques semaines à plusieurs années [8]. L'atteinte testiculaire se manifeste cliniquement par une réduction inconstante du volume testiculaire, parfois une réduction du volume de l'éjaculat, une oligospermie ou une azoospermie avec possibilité d'atteintes fonctionnelles du spermatozoïde mature, se traduisant notamment par des troubles de la mobilité [9].

Lors d'atteintes sévères, les lésions de la lignée germinale peuvent s'associer à une atteinte des cellules de Leydig. Une élévation des taux sériques de FSH et de LH est habituelle. Par contre, le taux de testostérone reste habituellement dans les limites de la normale grâce à une compensation d'origine surrénalienne [8].

\section{FERTILITE GLOBALE APRES CHIMIOTHERAPIE}

Une étude du National Cancer Institute portant sur 2283 adultes américains des deux sexes traités dans leur enfance pour cancer, a estimé la baisse de fertilité par rapport à une population témoin appariée à 15\% [6]. La fertilité était définie par la survenue d'au moins une grossesse après le mariage et l'étude ne tenait pas compte du type de chimiothérapie. La fertilité masculine était réduite de $24 \%$ alors que la fertilité féminine était inchangée. Les patients atteints de maladie de Hodgkin (déficit de $23 \%$ ) et de carcinome testiculaire (déficit $55 \%$ ) avaient une fertilité significativement diminuée par rapport au groupe contrôle, alors que celle des autres patients ne différait pas de celle de la population générale.

Avant la puberté, certaines drogues, principalement les agents alkylants, peuvent être responsables d'aménorrhée primaire par 
détériorations ovariennes massives [12]. Chez la femme, même si la procréation reste possible, la fenêtre de fertilité (période séparant la puberté de la ménopause) peut être réduite par la diminution du stock folliculaire $[5,8,20]$. Dans une série portant sur le pronostic gynécologique de patientes traitées par alkylants et radiothérapie entre 13 et 19 ans, le risque ménopause précoce était 27,4 fois plus élevé lorsque ces patientes atteignaient la tranche d'âge 21-25 ans par rapport à la population normale [5]. Ce risque diminuait à 4,6 fois pour la tranche d'âge 26-30 ans. Par ailleurs, l'exposition aux alkylants seuls conférait un risque relatif de ménopause précoce de 9,1 au patientes de 21-25 ans, contre 3,7 en cas de radiothérapie exclusive et de 1,1 après chirurgie seule. Ce risque était proportionnel aux doses administrées et à l'âge des patientes [5].

Chez l'homme, plus que le type de cancer, ce sont les médicaments utilisés et leurs dosages qui génèrent le risque de stérilité. En association une irradiation sous-diaphragmatique et une chimiothérapie sont fortement synergiques.

\section{ATTEINTE DE LA FERTILITE SELON LES DROGUES}

Les alkylants, puissants gonadotoxiques très largement utilisés en thérapeutique, sont responsables de la majorité des toxicités gonadiques constatées [6]. Les antimétabolites (méthotrexate, 5-fluorouracile 6-mercaptopurine, hydoxyurée) sont peu gonadotoxiques. Les antibiotiques sont rarement utilisés seuls mais ils ne semblent exercer aucune toxicité ovarienne significative [3]. Néanmoins la doxorubicine paraît potentialiser la gonadotoxicité de la cyclophosphamide. La mitomycine $\mathrm{C}$ occasionne des aménorrhées pour de faibles doses cumulées allant de 15 à $22 \mathrm{mg} / \mathrm{m}^{2}$ [22]. Aucune toxicité ovarienne n'est documentée avec l'actinomycine D administrée à des doses allant de 18 à $24 \mathrm{mg} / \mathrm{m}^{2}$. Parmi les poisons du fuseau, la vincristine lorsqu'elle est utilisée dans le traitement de la maladie de Hodgkin apparaît moins gonadotoxique que la vinblastine. La vinblastine peut induire des aménorrhées en association avec le cisplatine. Néanmoins, sa toxicité chez la femme est modeste. Chez l'homme, une oligospermie et une atteinte des cellules de Leydig a été documentée chez 6 hommes sur 8 traités pour lymphome et recevant $10 \mathrm{mg}$ de vinblastine par semaine [24]. L'étoposide (VP16) administré par voie orale est responsable d'oligoménorrhées ou d'aménorrhées persistant 4 à 6 mois après l'arrêt du traitement [26]. La toxicité ovarienne du cisplatine est bien documentée dans la combinaison PVB (cisplatine, vinblastine, bléomycine) utilisée pour le traitement des tumeurs germinales [1]. Les aménorrhées surviennent après une dose totale de $600 \mathrm{mg} / \mathrm{m}^{2}$ de cisplatine et sont toutes résolutives au plus tard 15 mois après la fin du traitement. La reprise des menstruations survient 2 à 18 mois après la fin du traitement.

Chez l'homme, la totalité des patients traités par cisplatine, doxorubicine, dacarbazine pour ostéosarcome a développée une azoospermie mais $78 \%$ des patients ont récupéré un spermogramme normal dans les 2 ans suivant la fin du traitement [17]. Seule la dose de cisplatine influençait cette récupération avec $43 \%$ d'évolutions favorables pour des doses supérieures à 600 $\mathrm{mg} / \mathrm{m} 2$ et $100 \%$ pour des doses inférieures avec un recul de 2 et 6 ans.

\section{ATTEINTE DE LA FERTILITE SELON LES PATHOLOGIES}

Au cours de maladie de la Hodgkin, chez la femme, les polychimiothérapies de type MOPP (méchloréthamine, vincristine, procarbazine, prednisolone) ou dérivées du MOPP sont responsables de troubles menstruels sévères avec environ $50 \%$ d'aménor- 
rhées et $35 \%$ de ménopauses précoces trois ans après le traitement [14]. Le risque de ménopause précoce après MVPP (méchloréthamine, vinblastine, procarbazine, prednisolone) est évalué à $80 \%$ avec un recul maximum de 7 ans [7]. Cet effet s'accroît avec l'âge des patientes lors du traitement et avec le nombre de cycles administrés. La fertilité des patientes conservant un cycle menstruel se révèle néanmoins normale avec un taux de conception compris entre 33 et $66 \%$ selon les séries [8]. Toutes patientes confondues ce taux est cependant réduit à $21 \%(7-29 \%)$ en moyenne avec un recul de 3 à 11 ans [14]. La chimiothérapie de type ABVD (doxorubicine, bléomycine, vinblastine, dacarbazine) ou VMB (vinblastine, méthotrexate, bléomycine) qui ne comporte pas d'agent alkylant ne s'avère pas avoir de conséquences ovariennes majeures en dehors d'une irrégularité menstruelle pendant la chimiothérapie et ne semble pas affecter la fertilité des patientes [13]. Chez l'homme, 20 à $30 \%$ des patients présentent des anomalies majeures de la spermatogenèse avant tout traitement, rendant l'estimation de la responsabilité de la chimiothérapie difficile [25]. Néanmoins, tous les patients indemnes d'anomalie du spermogramme au diagnostic récupèrent une spermatogenèse normale après VMB ou ABVD [3]. Par contre, la quasi totalité des patients traités par MOPP, gardent une oligospermie plus ou moins sévère associée à des troubles de la mobilité des spermatozoïdes [25]. Aucun des 117 patients traités par MVPP n'avait un spermogramme normal 1 à 8 ans après le traitement [25] et $16 \%$ gardaient une oligospermie sévère ou une azoospermie. Le MOPP serait légèrement moins toxique que le MVPP avec 14 à $22 \%$ de spermogramme normal 3 ans après le traitement [3].

Les données disponibles pour le cancer du sein concernent essentiellement les chimiothérapie de type CMF (cyclophosphamide, méthotrexate, fluorouracile). La toxicité de ce protocole est semblable à celle de la cyclophosphamide seule [7], 5-fluorouracile et méthotrexate n'exerçant qu'une toxicité mineure. Cent pour cent des femmes de moins de 30 ans développeront une aménorrhée pour une dose totale cumulée (DTC) de $20,8 \mathrm{~g} / \mathrm{m} 2$ de cyclophosphamide et après 30 ans pour une DTC de seulement $3,25 \mathrm{~g} / \mathrm{m} 2$.

Le choriocarcinome placentaire est très chimiosensible, et la fertilité publiée après de faibles doses de méthotrexate, 6-mercaptopurine, actinomycine $\mathrm{D}$, hydroxyurée, vincristine, vinblastine, ou de cyclophosphamide est bonne. Nous ne disposons pas de données suffisantes pour les sels de platine et pour la bléomycine. Le VP16 possède quant à lui une toxicité modérée mais certaine.

26 à $94 \%$ des patients atteints de cancer du testicule sont oligo ou azoospermiques au diagnostic, du fait de la tumeur. Chez 70 à $100 \%$ des patients, des anomalies sévères du spermogramme persistent jusqu'à 12 à 18 mois après le traitement [10]. Une récupération plus ou moins complète survient habituellement dans les 3 ans, que le protocole thérapeutique inclut ou non du cisplatine. Après PVB (cisplatine, vinblastine, bléomycine) de 35 à $40 \%$ des patients restent fertiles à en juger par les données du spermogramme [21].

Plusieurs courtes séries rapportent des grossesses après traitement pour leucémie aiguë, essentiellement après leucémie aiguë lymphoblastique (LAL). La fertilité après traitement combinant vincristine, anthracyclines, 6-mercaptopurine, méthotrexate et corticoïdes est habituellement conservée [11]. L'utilisation d'agents alkylants ou de cytosine arabinoside à plus de $1 \mathrm{~g} / \mathrm{m}^{2}$ est responsable de toxicité gonadique dans les deux sexes dont le retentissement est difficile à chiffrer précisément en raison de la grande variation protocoles schémas thérapeutiques actuels et du manque de recul pour évaluer la toxicité gonadique chez des patients pédiatriques [11]. 
Trente sept grossesses ont été rapportées chez 27 femmes âgées de 17 à 36 ans, traitées par allogreffe de moelle osseuse pour aplasie médullaire ( 15 femmes) ou pour leucémie aiguë (12 femmes)[16]. Toutes avaient reçu une conditionnement par cyclophosphamide seule à forte dose (120 $\mathrm{mg} / \mathrm{kg}$ ) (15 femmes), ou combinée à une irradiation corporelle totale ( 10 femmes) ou à du melphalan ( 2 femmes). Les grossesses sont survenues dans un délai de 8 à 96 mois après la greffe. Après cyclophosphamide seule, 18 grossesses chez 15 femmes ont permis la naissance de 13 enfants vivants contre 5 avortements ( 3 spontanés et 2 thérapeutiques). Trois grossesses ont été menées à terme près cyclophosphamidemelphalan. Après irradiation corporelle totale, 12 grossesses dont 1 en cours, 6 enfants vivants et 5 avortements ( 4 spontanés et 1 thérapeutique) sont rapportées. Deux grossesses sont survenues chez des femmes aménorrhéiques, sans aide de fécondation in vitro.

\section{PRONOSTIC DE LA GROSSESSE}

Des preuves biologiques attestent l'effet mutagène de la chimiothérapie. Chez l'animal l'ABVD (doxorubicine, bléomycine, vinblastine, dacarbazine) et le thiotépa induisent des cassures et des translocations chromosomiques ainsi que des aneuploïdies dans la lignée spermatique [8]. Des cassures chromosomiques et des échanges de chromatides sœurs ont également été mis en évidence dans les lymphocytes du sang périphérique de femmes traitées pour choriocarcinome placentaire, leucémie aiguë et tumeurs pédiatriques [in 8]. Néanmoins, ces anomalies régressaient dans les deux ans suivant l'arrêt du traitement. Malgré les preuves biologiques du pouvoir mutagène de la chimiothérapie, aucune série ne montre d'élévation significative des anomalies congénitales chez les enfants nés de parents exposés à des agents antimitotiques. Une étude portant sur 94 enfants issus de femmes traitées pour choriocarcinome placentaire retrouve une incidence des anomalies chromosomiques congénitales de $0,7 \%$ contre $1,6 \%$ dans la population témoin et aucun retard mental avec un recul maximal de 25 ans [23]. Une seule publication portant sur 202 grossesses, chez 100 patients antérieurement traités par chimiothérapie, a rapporté deux anomalies cardiaques après actinomycine $\mathrm{D}$ (une tétralogie de Fallot et une anomalie du septum ventriculaire) [11]. Néanmoins cette observation n'a pas été confirmée. Une étude cas témoin récente comparant 45200 mères et 41158 pères d'enfants porteurs d'anomalies congénitales à une population contrôle appariée a retrouvé un nombre équivalent de patients antérieurement traités pour cancer dans les deux groupes [25]. Le risque de donner naissance à un enfant anormal après avoir reçu une chimiothérapie n'apparaît donc pas supérieur à celui de la population générale qui est estimé à $3 \%$ [in 19]. En outre, dans cette étude aucun lien n'a pu être établi entre un médicament et une malformation donnée, notamment entre une anomalie cardiaque et l'actinomycine D. Certaines études ont montré un excès d'avortements ou de naissances prématurées chez des patientes traitées pour cancer, mais l'ont expliqué par l'existence d'anomalies utérines indépendantes ou secondaires à la pathologie ou à une irradiation [15].

Plusieurs mécanismes ont été proposés pour tenter d'expliquer l'absence de tératogénicité de la chimiothérapie en pratique clinique. Chez l'homme, les cellules les plus matures de la lignée spermatique seraient soit tuées par l'exposition à certains agents cytotoxiques comme la mitomycine $\mathrm{C}$, soit mutées, les cellules mutées étant incapables de fertilisation. Des réparations de lésions chromosomiques spermatiques ont été démontrées chez la souris. Enfin un arrêt de maturation de cellules souches spermatiques affectées par une atteinte génétique a été suspectée. Chez la femme, 
l'atteinte concomitante des cellules thécales a été décrite comme pouvant affecter le développement d'un œuf atteint [in 8].

\section{CANCEROGENESE DANS LA DESCENDANCE}

Une étude récente a montré que seuls les cancers avec une transmission familiale prouvée, comme le rétinoblastome ou la tumeur de Wilms, sont retrouvés avec une fréquence accrue dans la descendance d'anciens cancéreux atteints de la même tumeur [18]. Pour les autres tumeurs, la descendance ne semble pas présenter un risque accru par rapport à la population générale. Néanmoins, les enfants n'étant âgés que de 11 ans en moyenne lors de l'étude, ce risque n'est pas totalement écarté et réclame de la vigilance.

\section{CONSEILS AUX PATIENTS ET AUX CLINICIENS}

Toute chimiothérapie ne s'accompagnant pas de stérilité, le pronostic gonadique ne peut être toutefois établi qu'en fonction du sexe des patients, de la pathologie traitée (baisse de fertilité au cours de la maladie de Hodgkin ou du cancer du testicule), des drogues employées, de leur doses, et de l'âge des patient(e)s. Le pronostic gonadique devrait, dans la mesure du possible, être expliqué aux patient(e)s, ou aux parents des enfants atteints de tumeur maligne, dès le début de la maladie. En fait bien souvent, ce problème est relégué au second plan, soit par oubli de la part du médecin, soit ignoré par le patient du fait du choc émotionnel lié à l'annonce du diagnostic. La fertilité future est une question d'autant plus importante à aborder que la discussion devra obligatoirement envisager l'opportunité d'une cryoconservation de sperme avant toute chimiothérapie, chez les hommes en âge et désireux de procréer, lorsque le traitement risque d'avoir des effets délétères sur la fonction testiculaire.

Même lorsqu'un des membres du couple soumis à une chimiothérapie garde soit des perturbations du cycle menstruel soit une oligo-azoospermie, tout risque de grossesse ne peut être écarté en raison du risque d'ovulation inopinée lors d'une aménorrhée apparemment installée [16], de récupération tardive de la fonction spermatocytaire, ou de la possibilité de procréation même en cas d'oligospermie sévère [19]. Les couples ne souhaitant pas avoir d'enfant doivent donc être avertis de la nécessité d'une contraception.

Les couples désirant une grossesse doivent être rassurés quant au risque de tératogénicité et de cancer induit dans la descendance, dans la limite des connaissances actuelles. Cette opinion, doit être néanmoins nuancée lorsque la tumeur traitée est à composante familiale possible (tumeur de Wilms, rétinoblastome). Un caryotype à la recherche d'une monosomie 11p- ou 13qdoit alors être effectuée [19]. Dans les autres situations, le caryotype n'est d'aucune utilité. L'utilisation d'une drogue de développement récent pour laquelle les données sont insuffisantes doit également amener à formuler des réserves.

La date de la grossesse chez une femme antérieurement traitée par chimiothérapie doit être discutée en fonction du pronostic de la tumeur, de sa compatibilité avec une grossesse (cancer du sein). En général un délai de trois ans est requis entre la fin du traitement et le début d'une grossesse. $\mathrm{Ce}$ délai ne doit pas être exagérément augmenté, à cause du risque de ménopause précoce induit par le traitement.

Lorsqu'une grossesse désirée ne peut être obtenue après les délais habituels et lorsqu'un cycle menstruel persiste chez la femme, une prise en charge spécialisée en vue d'une éventuelle fécondation in vitro doit être envisagée [8]. 


\section{CONCLUSION}

La connaissance de la gonadotoxicité de chaque drogue au même titre que les autres toxicités viscérales est indispensable. Elle permet de fournir au patient des renseignements fonctionnels légitimes, mais permet également le choix d'une combinaison thérapeutique. Avec le développement de traitements performants les avancées thérapeutiques visent non seulement à améliorer le contrôle de la maladie mais également à minimiser la toxicité, en particulier la gonadotoxicité. A efficacité égale, on préférera le traitement le moins toxique (par exemple ABVD ou MOPP/ABVD sera préféré au MOPP dans le traitement de la maladie de Hodgkin)[3].

\section{REFERENCES}

1. BAKRI YN, GIVEN FT. : Normal pregnancy and delivery following conservative surgery and chemotherapy for ovarian endodermal sinus tumor. Gynecol Oncol 1984, 19 : 222-225.

2. BLEYER WA. : The impact of childhood cancer in the United States and the world. CA Cancer J Clin $1990,40: 355-367$.

3. BONNADONNA G, SANTORO A, VIVIANI S, VALAGUSSA P. : Treatment strategies for Hodgkin's disease. Seminar Hematol 1988, 25 : 51-57.

4. BRENNER J, VUGRIN D, WHITMORE WF. : Effect of treatment on fertility and sexual function in males with metastatic nonseminomatous germcell tumors of testes. Am J Clin Oncol 1985, 8 : 178-182.

5. BYRNE J, FEARS FR, GAIL MG, PEE D, CONNELLY RR, AUSTIN DF. : Early menopause in long-term survivors of cancer during adolescence. Am J Obstet Gynecol 1992, 166 : 788793.

6. BYRNE J, MULVIHILL JJ, MYERS MH,ET AL. : Effects of treatment on fertility in long-term survivors of childhood or adolescent cancer. N Engl $J$ Med 1987, 317 : 1315-1381.

7. CADMANABHAN N, WANG DY, MOORE JW, RUBENS RD. : Ovarian function and adjuvant chemotherapy for early breast cancer. Eur J Cancer Clin Oncol 1987, $23: 745-748$.
7'. CHAPMAN RM. :Gonadal toxicity and teratogenicity. In Perry MC ed. The chemotherapy source book. Williams and Wilkins. Baltimore. 1992, pp 710-753.

8. CURTIN JP, ADCOCK I.L. : Pregnancy following treatment of endodermal sinus tumor of the ovary with combination chemotherapy including cis-platinium. Gynecol Oncol 1986, $24: 268-270$.

9. DRASGA RE, EINHORN LH, WILLIAMS SD, PATEL DN, S'TEVENS EE. : Fertility after chemotherapy for testicular cancer. J Clin Oncol 1983,1 : 179-183.

10. GREEN DM, HALI. B, ZEVON MA. : Pregnancy outcome after treatment for acute lymphoblastic leukemia during childhood or adolescence. Cancer $1989,64: 2335-2339$.

11. HIMELSTEIN-BRAW R, PETERS H, FABER M. : Morphological study of the ovaries of leukaemic children. Br J Cancer 1978, 38 : 82-87.

12. HORNING SJ, HOPPE RT, HANCOCK SL, ROSENBERG SA. :Vinblastine, bleomycin, and methotrexate. An effective adjuvant in favorable Hodgkin's disease. J Clin Oncol 1988, 6 : 18221831.

13. HORNING SJ, HOPPE RT, KAPLAN HS, ROSENBERG SA. : Female reproductive potential after treatment for Hodgkin's disease. N Engl J Med 1981, 304 : 1377-1382.

14. LI FP, GIMBERE K, GELBER RD, ET AL. : Outcome of pregnancy in survivors of Wilm's tumor. JAMA 1987, $257: 216-219$.

15. LIPTON JH, DERZKO C, FYLES G, MEHARCHAND J, MESSNER HA. : Pregnancy after bone marrow transplantation: three case reports. Bone Marrow Transplantation 1993, $11: 415$ 418.

16. MEISTRICH ML, CHAWLA SP, DA CUNHA MF, JOHNSON SL, PLAGER C, PAPADOPOULOS NE, LIPSCHUIZ LP, BENJAMIN RS. : Recovery of sperm production after chemotherapy for osteosarcoma. Cancer 1989, $63: 2115-2123$.

17. MULVIHILL JJ, MYERS MH, CONNELLY RR ET AL. :Cancer in offspring of long-term survivors of childhood and adolescence cancers. Lancet 1987, ii : $813-817$.

18. NICHOLSON HS, BYRNE J. : Fertility and pregnancy after treatment for cancer during childhod or adolescence. Cancer 1993, 71 : 33923399 .

19. NICOSIA SV, MATUS-RIDLEY M, MEADOWS AT. :Gonadal effects of cancer therapy in girls. Cancer 1985, 55 : 2364-2372. 
20. ROTH BJ, GREIST A, KUBILIS PS, WILLIAMS SD, EINHORN LH. : Cisplatin-based combination chemotherapy for disseminated germ-cell tumors : long-term follow-up. J Clin Oncol 1988, 6 :12391247.

21. SCHILSKY RL, YARBRO JW. : Pharmacology of antineoplastic drugs. In: Perry MC, Yarbro JW eds. Toxicity of chemotherapy. Grune and Stratton. Orlando. 1984, pp 21-59.

22. SONG H, WU P, WANG Y, YANG X, DONG S. : Pregnancy outcomes after successful chemotherapy for choriocarcinoma and invasive mole: longterm follow-up. Am J Obstet Gynecol 1988, 158 : 538-545.

23. VILAR O. : Effect of cytostatic drugs on human testicular function. In: Mancini RE, Martini L eds. Male fertility and sterility. Academic Press. New York. 1974, pp 423-440.

24. WHITEHEAD E, SHALET SM, BLACKLEDGE G, TODD I, CROWTHER D, BEARDWELL CG. : The effect of Hodgkin's disease and combination chemotherapy on gonadal function in the adult male. Cancer $1982,49: 418-422$.

25. WONG LC, CHOO YC, MA HK. : Primary oral etoposide therapy in gestational trophoblastic disease. Cancer 1986, $58: 14-17$.

\section{ABSTRACT \\ Testicular and ovarian function after chemotherapy \\ C. Linassier, Ph. Colombat}

Recent advances in the treatment of cancer now permits long-term remissions and can cure a large amount of young. It is now well established that infertility and premature menopause are common consequences of successeful treatment. Much is known about the fertility and sexual effects of chemotherapy and allow development of new strategies to reduce infertilty.
Alkylating agents are among the most commonly used chemotherapy agents and are for the most part highly gonadotoxic. Other drugs like cisplatin, vinca alkaloids, epipodophyllotoxins and antibiotics have moderate intrinsic toxicity whereas antimetabolites exert little if any toxic effect. Nevertheless, drugs are often used in combinations which are more toxic than single agents. Gonadal toxicity is usually dose dependant. Infertility is more frequent in males than in females and prepubertal girls have better prognosis though prepubertal ovaries are profoundly affected by chemotherapy. The onset of chemotherapy-related ovarian failure is age related but the risk of premature menopause still exists in women who recover menstrual cycles after amenorrhea. Fertility is usually worse after Hodgkin's disease and testes cancer than after other cancers. No teratognesis in the offspring was published so far after chemotherapy. Nevertheless, men and women should be expressly discouraged from being party to a conception while they are receiving cancer therapy. Adults surviving to cancer should wait at least two years after completion of therapy before attempting parenthood.

Key words : Testes, ovary, fertility, chemotherapy, cancer, leukemia

Note de la rédaction : Voir Andrologie, Vol. 5, $\mathrm{n}^{\circ} 4$, décembre 1995. Ce numéro est en grande partie consacré aux effets des traitements anticancéreux sur reproduction et sexualité. 